\title{
Dynamic Model of an Ammonia Synthesis Reactor based on Open Information
}

\author{
Asanthi Jinasena Bernt Lie Bjørn Glemmestad \\ Faculty of Technology, University College of Southeast Norway, Norway, \\ \{asanthi.jinasena, Bernt. Lie\}@usn.no
}

\begin{abstract}
Ammonia is a widely used chemical, hence the ammonia manufacturing process has become a standard case study in the scientific community. In the field of mathematical modeling of the dynamics of ammonia synthesis reactors, there is a lack of complete and well documented models. Therefore, the main aim of this work is to develop a complete and well documented mathematical model for observing the dynamic behavior of an industrial ammonia synthesis reactor system. The model is complete enough to satisfactorily reproduce the oscillatory behavior of the temperature of the reactor.
\end{abstract}

Keywords: modeling, ammonia, reactor, dynamic, simulation

\section{Introduction}

The control of the ammonia synthesis reactor is an interesting topic in the industrial and scientific community, because of the importance and the dynamics of it. Mathematical modeling of the ammonia synthesis loop is a common strategy for understanding and controlling these dynamics. Most of the studies are focused on steady state operation. Simulation of ammonia synthesis reactors for design, optimization (Baddour et al., 1965; Murase et al., 1970; Singh, 1975) and control (Shah, 1967; Singh and Saraf, 1979) has been reported since the late 1960s. However, studies on reactor instability started a few years earlier (van Heerden, 1953). A few studies have been done on dynamic modeling of ammonia synthesis reactors. However, most available models are incomplete in information: missing parameter values, missing or incorrect units, missing expressions for reaction rate due to confidentiality, inconsistent thermodynamics and missing operating conditions. The main objective of this study is therefore to compile a complete, well-documented and easily accessible dynamic model purely based on information available through open publications. The model is used to reproduce the oscillatory behavior of temperature which has been reported especially on manually controlled industrial reactors (Naess et al., 1993; Morud and Skogestad, 1993; Morud, 1995; Morud and Skogestad, 1998; Rabchuk et al., 2014; Rabchuk, 2014).

Considering the few dynamic models that have been reported, Naess et al. (1993) developed a model for op- timization and control of the ammonia synthesis process based on an incident of an ammonia synthesis plant in Germany. The simulations were verified using the plant data. For the same incident, Morud (1995), Morud and Skogestad $(1993,1998)$ analyzed the instability through a dynamic model to reproduce the behavior of rapid temperature oscillations observed in the industrial ammonia reactor system by stepping down the reactor pressure. A linear dynamic analysis was done on the model. It has been shown that the cause of the limit cycle behavior of the reactor was positive temperature feedback from the heat exchanger and a non-minimum phase behavior of the temperature response. A feedback controller is suggested to control this behavior (Morud and Skogestad, 1998).

Rabchuk (2014) and Rabchuk et al. (2014) have developed a dynamic model for testing the stability of an industrial ammonia synthesis reactor. The system consisted of a catalytic bed ammonia synthesis reactor and a heat exchanger and the oscillations were obtained by stepping down the feed temperature. A stability analysis was also done for selected process parameters (Rabchuk, 2014).

This paper consists of a detailed model description in Section 2, including the assumptions, the topology and the descriptive model development for the reactor and the heat exchanger. This is followed by the simulation results and discussion with a comparison with previous work in Section 3. All the values and units for the used parameters and operating conditions are included in the Appendix.

\section{Mathematical Model}

The Haber-Bosch process is used to produce ammonia from the following reaction using an iron based catalyst,

$$
\mathrm{N}_{2(\mathrm{~g})}+3 \mathrm{H}_{2(\mathrm{~g})} \stackrel{\mathrm{Fe}}{\rightleftharpoons} 2 \mathrm{NH}_{3(\mathrm{~g})} .
$$

Argon (Ar) is also present as an inert gas. The ammonia synthesis process includes catalytic bed reactors for ammonia formation with heat exchangers, where the product gas streams are cooled by the feed gas streams. A simplified diagram of the reactor configuration is shown in Figure 1. The reactor is considered to have one fixed catalytic bed and no bypass or intermediate cooling gas streams for simplicity. The heat exchanger is considered to be a simple counter current heat exchanger.

In this system, $\dot{m}_{i}$ and $\dot{m}_{o}$ are the inlet and outlet mass flow rates of the system, respectively. $T_{i}$ is the temperature 


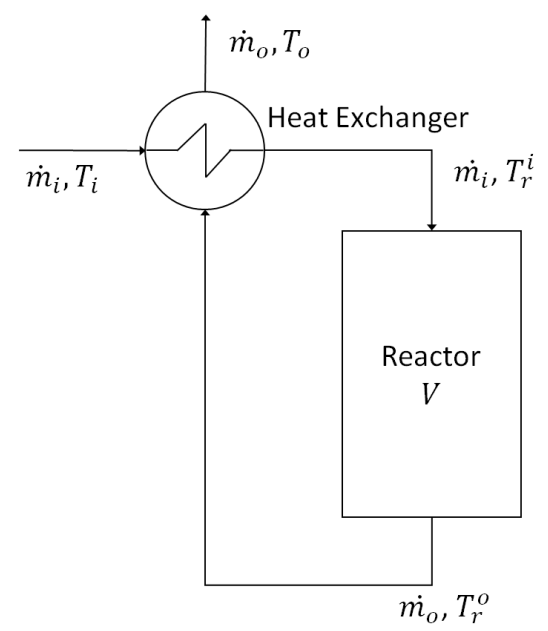

Figure 1. A simplified reactor configuration.

of the inlet flow of the heat exchanger. $T_{o}$ is the temperature of the flow out of the heat exchanger, $T_{r}^{i}$ is the temperature of the reactor inlet and $T_{r}^{o}$ is the temperature of the reactor outlet. The volume of the reactor is denoted by $V$. The input $\dot{m}_{i}$ and the set point to the reactor pressure controller can be manipulated. $T_{i}$ and the inlet mole fractions of various species $\left(x_{j}^{i}\right)$ are considered as disturbances to the system. The temperature of the reactor $T_{r}$ is the output of interest.

\subsection{Assumptions}

The following assumptions are used:

- The model is one-dimensional, i.e. the temperature and molar gradients only vary in the axial direction.

- The Temkin-Pyzhev reaction rate expression is valid for the system (Murase et al., 1970; Morud and Skogestad, 1998; Froment et al., 2010).

- The discretized reactor volume compartments are well mixed.

- No heat or mass diffusion in the system.

- Individual gases and gas mixture behave as ideal gas.

- The catalyst activity is uniform throughout the reactor.

- The heat transfer coefficient, heat of reaction and heat capacities are constants.

- Reactor pressure is controlled perfectly.

\subsection{Development of Model}

\subsubsection{Material Balance}

The pressure inside the reactor $(p)$ is considered to be constant. The schematics shown in Figure 2 depicts the distributed reactor model. For volume compartment $V_{1}$, the

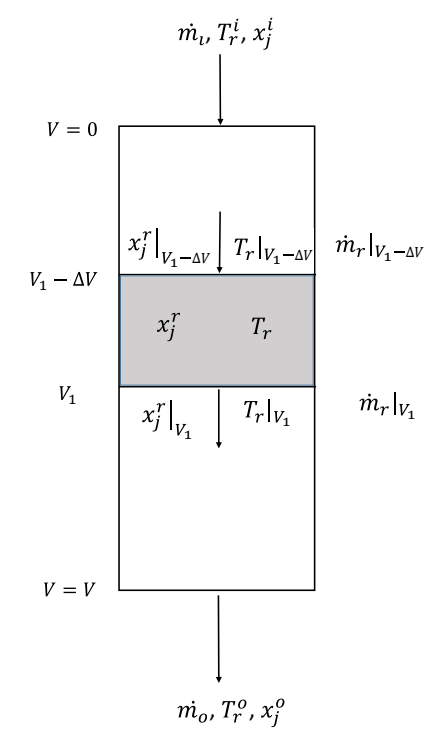

Figure 2. A schematic diagram of the distributed reactor model.

mole balance equation can be written as shown in Eq. 2 .

$$
\left.\frac{d}{d t} n_{j}^{r}\right|_{V_{1}}=\left.\dot{n}_{j}^{r}\right|_{V_{1}-\Delta V}-\left.\dot{n}_{j}^{r}\right|_{V_{1}}+\left.\dot{n}_{j}^{r, g}\right|_{V_{1}}
$$

Here, $n_{j}^{r}$ is the number of moles inside the reactor compartment at a given time $t, \dot{n}_{j}^{r}$ is the rate of moles leaving the reactor compartment and $\dot{n}_{j}^{r, g}$ is the rate of generation of moles inside the reactor compartment. Superscript $r$ denotes the reactor and subscript $j$ denotes the particular specie, where $j \in\left(\mathrm{H}_{2}, \mathrm{~N}_{2}, \mathrm{NH}_{3}, \mathrm{Ar}\right)$. The rate of generation can be expressed using the reaction rate $r$, stoichiometric matrix $v$ and the catalyst mass $m_{c}$ in the reactor volume,

$$
\left.\dot{n}_{j}^{r g}\right|_{V_{1}}=\left.v_{j} r m_{c}\right|_{V_{1}}=\left.v_{j} r\right|_{V_{1}} m_{c} \frac{\Delta V}{V}
$$

where $v=\left[\begin{array}{llll}-3 & -1 & 2 & 0\end{array}\right] . \Delta V$ is the volume of a reactor compartment. The rate of reaction (rate of Nitrogen consumption per unit catalyst mass) can be found using the Temkin-Pyzhev equation (Murase et al., 1970; Morud and Skogestad, 1998).

$$
\left.r\right|_{V_{1}}=\left.\frac{f}{\rho_{c}}\left(k_{+} \frac{p_{N_{2}} p_{H_{2}}{ }^{1.5}}{p_{N H_{3}}}-k_{-} \frac{p_{N H_{3}}}{p_{H_{2}}{ }^{1.5}}\right)\right|_{V_{1}}
$$

where $f$ is the catalyst activity factor, $\rho_{c}$ is the packing density of the catalyst, $k_{+}$and $k_{-}$are the rate constants of the forward and reverse reactions, respectively, and $p_{j}$ denotes the partial pressure of the species in the reactor compartment. Using the Gibbs Free Energy approach at a constant temperature, the reverse reaction rate can be expressed as follows (Froment et al., 2010),

$$
k_{-}=k_{0}^{-} \exp \left(-\frac{E_{-}}{R T}\right) \text {. }
$$


Using the chemical kinetics of the reaction, $k_{+}$can be found from the equilibrium constant, $K_{p}$,

$$
k_{+}=k_{-} K_{p} .
$$

The value for $K_{p}$ can be computed using available correlations. The Gillespie-Beattie correlation is selected as the most suitable correlation for this system (Gillespie and Beattie, 1930). The correlation is

$$
K_{p}^{G B}=K_{p}^{G B *} 10^{\alpha \cdot p^{*}},
$$

where $K_{p}^{G B}$ is the Gillespie-Beattie equilibrium constant. The pressure correction coefficient $\alpha$ is given as

$$
\alpha=\frac{0.1191849}{\left.T_{r}\right|_{V_{1}}}+\frac{91.87212}{\left.T_{r}\right|_{V_{1}} ^{2}}+\frac{25122730}{\left.T_{r}\right|_{V_{1}} ^{4}},
$$

where $T_{r}$ is the temperature of the reactor compartment. The value of the $K_{p}^{G B *}$ can be computed from Eq. 9.

$$
\begin{aligned}
\log K_{p}^{G B *}= & -\left.2.69112 \log T_{r}\right|_{V_{1}}-5.51926 \times\left. 10^{-5} T_{r}\right|_{V_{1}} \\
& +1.84886 \times\left. 10^{-7} T_{r}\right|_{V_{1}} ^{2}+\frac{2001.6}{\left.T_{r}\right|_{V_{1}}} \\
& +2.6899
\end{aligned}
$$

The dimensionless pressure $p^{*}$ is

$$
p^{*}=\frac{p}{p^{\sigma}},
$$

where $p^{\sigma}$ is the atmospheric pressure in the given pressure unit. The relationship between the two rate coefficients is given by Eq. 11 .

$$
K_{p}=K_{p}^{G B^{2}}
$$

Temperature $T_{r}$ at reactor compartment $V_{1}$ can be found by rearranging the ideal gas law to express the temperature as shown in Eq. 12.

$$
\left.T_{r}\right|_{V_{1}}=\frac{p \cdot \Delta V}{\left.n_{r}\right|_{V_{1}} R} \varepsilon
$$

Here, $\varepsilon$ is the void fraction of the catalyst and $n_{r}$ is the total number of moles in the reactor volume where

$$
n_{r}=\sum_{j} n_{j}^{r},
$$

and $R$ is the universal gas constant.

\subsubsection{Energy Balance}

The energy balance equation for volume compartment $V_{1}$ is

$$
\begin{aligned}
\left.\frac{d}{d t}(H-p V)\right|_{V_{1}}= & \left.\dot{H}\right|_{V_{1}-\Delta V}-\left.\dot{H}\right|_{V_{1}} \\
& +\left.\dot{Q}\right|_{V_{1}}+\left.\dot{W}\right|_{V_{1}} .
\end{aligned}
$$

Assuming no heat flow $\dot{Q}$, no shaft work $\dot{W}$ and constant pressure, the Eq. 14 can be simplified into

$$
\left.\frac{d}{d t} H\right|_{V_{1}}=\left.\dot{H}\right|_{V_{1}-\Delta V}-\left.\dot{H}\right|_{V_{1}},
$$

where $H$ is the enthalpy of the reactor volume at a given time $t, \dot{H}$ is the rate of enthalpy of the flow into/out of the reactor volume. $H$ can be written using the enthalpies of individual components of the mixture,

$$
\left.H\right|_{V_{1}}=\left.\sum_{j} n_{j}^{r} \tilde{H}_{j}\right|_{V_{1}}+\left.m_{c} \hat{H}_{c}\right|_{V_{1}}
$$

where $\tilde{H}_{j}$ is the molar enthalpy of pure gas $j \in$ $\left(\mathrm{H}_{2}, \mathrm{~N}_{2}, \mathrm{NH}_{3}, \mathrm{Ar}\right)$ and $\hat{H}_{c}$ is the specific enthalpy of the catalyst. Similarly $\dot{H}$ is

$$
\left.\dot{H}\right|_{V_{1}}=\left.\sum_{j} \dot{n}_{j}^{r} \tilde{H}_{j}\right|_{V_{1}} .
$$

Using Eqs. 2, 16 and 17, Eq. 15 can be developed as follows,

$$
\begin{aligned}
& \left.\sum_{j} n_{j}^{r} \frac{d \tilde{H}_{j}}{d t}\right|_{V_{1}}+\left.m_{c} \frac{d \hat{H}_{c}}{d t}\right|_{V_{1}}=-\left.\sum_{j} \dot{n}_{j}^{r g} \tilde{H}_{j}\right|_{V_{1}} \\
& +\left.\sum_{j} \dot{n}_{j}^{r}\right|_{V_{1}-\Delta V}\left(\left.\tilde{H}_{j}\right|_{V_{1}-\Delta V}-\left.\tilde{H}_{j}\right|_{V_{1}}\right) .
\end{aligned}
$$

With the use of following approximations,

$$
\begin{aligned}
d \tilde{H}_{j} & \approx \tilde{c}_{p, j} d T \\
\tilde{H}_{1}-\tilde{H}_{2} & \approx \bar{c}_{p}\left(T_{1}-T_{2}\right)
\end{aligned}
$$

where $\tilde{c}_{p, j}$ is molar heat capacity of each gas and $\overline{\tilde{c}}_{p}$ is the average molar heat capacity of the gas mixture, the model can be simplified into

$$
\begin{aligned}
\left.C_{p} \frac{d T_{r}}{d t}\right|_{V_{1}}= & \left.\dot{n}_{r} \overline{\tilde{c}}_{p}\right|_{V_{1}-\Delta V}\left(\left.T_{r}\right|_{V_{1}-\Delta V}-\left.T_{r}\right|_{V_{1}}\right) \\
& -\left.\Delta \tilde{H}_{r} r m_{c}\right|_{V_{1}},
\end{aligned}
$$

where $\Delta \tilde{H}_{r}$ is the heat of reaction. Here, $C_{p}$ is the heat capacity of the reactor compartment,

$$
C_{p}=\sum_{j} n_{j}^{r} \tilde{c}_{p, j}+m_{c} \hat{c}_{p, c},
$$

where $\hat{c}_{p, c}$ is the specific heat capacity of the catalyst.

Taking the time derivative of ideal gas law with constant pressure and then substituting the expression in Eq. 21 for the term $\frac{d T_{r}}{d t}$ will lead to the Eq. 23,

$$
\begin{gathered}
\left.\dot{n}_{r}\right|_{V_{1}}=\left.\dot{n}_{r}\right|_{V_{1}-\Delta V}+\left.\dot{n}^{r, g}\right|_{V_{1}}+\left.\frac{n_{r}}{T_{r} C_{p}}\right|_{V_{1}}\left[\left.\dot{n}_{r} \overline{\tilde{c}}_{p}\right|_{V_{1}-\Delta V}\right. \\
\left.\left(\left.T_{r}\right|_{V_{1}-\Delta V}-\left.T_{r}\right|_{V_{1}}\right)-\left.\Delta \tilde{H}_{r} r m_{c}\right|_{V_{1}}\right]
\end{gathered}
$$


which can be re-arranged into

$$
\begin{aligned}
\left.T_{r} C_{p} \dot{n}^{r, g}\right|_{V_{1}}- & \left.\Delta \tilde{H}_{r} n_{r} r m_{c}\right|_{V_{1}}=\left.T_{r} C_{p} \dot{n}_{r}\right|_{V_{1}} \\
& -\left.\dot{n}_{r}\right|_{V_{1}-\Delta V}\left[\left.T_{r} C_{p}\right|_{V_{1}}+\left.\left.\overline{\tilde{c}}_{p}\right|_{V_{1}-\Delta V} n_{r}\right|_{V_{1}}\right. \\
& \left.\left(\left.T_{r}\right|_{V_{1}-\Delta V}-\left.T_{r}\right|_{V_{1}}\right)\right]
\end{aligned}
$$

This can be written in matrix form,

$$
b=A \cdot \dot{n}_{r}
$$

where $A \in \mathbf{R}^{N \times N}$ and $i_{r}, b \in \mathbf{R}^{N \times 1}$. Here $N$ is the number of reactor compartments in the reactor. If all compartments are considered to have equal volumes of $\Delta V$ with $N=\frac{V_{r}}{\Delta V}$, then

$$
\begin{aligned}
b_{1}= & \left.T_{r} C_{p} \dot{n}^{r, g}\right|_{\Delta V}-\left.\Delta \tilde{H}_{r} n_{r} r m_{c}\right|_{\Delta V}+\left.\dot{n}_{r}\right|_{0} \\
& {\left[\left.T_{r} C_{p}\right|_{\Delta V}+\left.\left.\dot{n}_{r} \overline{\tilde{c}}_{p}\right|_{0} n_{r}\right|_{\Delta V}\left(\left.T_{r}\right|_{0}-\left.T_{r}\right|_{\Delta V}\right)\right] } \\
b_{i}= & \left.T_{r} C_{p} \dot{n}^{r, g}\right|_{i \Delta V}-\left.\Delta \tilde{H}_{r} n_{r} r m_{c}\right|_{i \Delta V}, \\
& i \in\{2,3, \ldots, N\}
\end{aligned}
$$

and,

$$
\begin{aligned}
A_{i, i} & =\left.T_{r} C_{p}\right|_{i \Delta V}, \quad i \in\{1,2, \ldots, N\} \\
A_{i, i-1} & =-\left.T_{r} C_{p}\right|_{i \Delta V}-\left.\left.\overline{\tilde{c}}_{p}\right|_{(i-1) \Delta V} n_{r}\right|_{i \Delta V} \\
& \left(\left.T_{r}\right|_{(i-1) \Delta V}-\left.T_{r}\right|_{i \Delta V}\right), i \in\{2,3, \ldots, N\} .
\end{aligned}
$$

Solving Eq. 25 gives $\dot{n}_{r}$, and then $\dot{n}_{j}^{r}$ can be found from

$$
\left.\dot{n}_{j}^{r}\right|_{V_{1}}=\left.x_{j}^{r} \dot{n}_{r}\right|_{V_{1}} .
$$

Here, $x_{j}^{r}$ is the mole fraction, which can be found using the mole numbers.

$$
\left.x_{j}\right|_{V_{1}}=\frac{\left.n_{j}\right|_{V_{1}}}{\left.\sum_{j} n_{j}\right|_{V_{1}}}
$$

\subsubsection{Heat Exchanger}

The heat exchanger is considered as a standard countercurrent heat exchanger with steady state heat transfer. The energy balance equation can be written as

$$
\frac{d T_{c}}{d x}=\frac{U A}{\dot{m}_{i} \hat{c}_{p}^{i} L}\left(T_{h}-T_{c}\right),
$$

and

$$
\frac{d T_{h}}{d x}=\frac{U A}{\dot{m}_{o} \hat{c}_{p}^{o} L}\left(T_{h}-T_{c}\right)
$$

where $T_{h}, T_{c}$ are the temperatures of hot (outlet stream of the heat exchanger) and cold (inlet stream of the heat exchanger) streams at time $t$, respectively. $U$ is the overall heat transfer coefficient of the heat exchanger and $A$ is the total heat transfer area of the heat exchanger and $\hat{c}_{p}^{i}$ and $\hat{c}_{p}^{o}$ are the specific heat capacities of the inlet and outlet gas mixtures, respectively. $L$ is the length of the heat exchanger and $x$ is the position along the heat exchanger where $x=[0, L]$.

Assuming that $\dot{m}_{o} \hat{c}_{p}^{o}$ and $\dot{m}_{i} \hat{c}_{p}^{i}$ have the same values, and $\frac{U A}{\dot{m}_{i} \hat{c}_{p}^{i}}$ is independent of $x$, Eqs. 32 and 33 can be simplified further to give an explicit expression for the reactor inlet temperature as

$$
T_{r}^{i}=\frac{T_{i}+\frac{U A}{\dot{m}_{i} \hat{c}_{p}^{i}} T_{r}^{o}}{1+\frac{U A}{\dot{m}_{i} \hat{c}_{p}^{i}}} .
$$

Similarly, the expression for the outlet temperature of the heat exchanger is

$$
T_{o}=\frac{T_{r}^{o}+\frac{U A}{\dot{m}_{i} \hat{c}_{p}^{i}} T_{i}}{1+\frac{U A}{\dot{m}_{i} \hat{c}_{p}^{i}}} .
$$

\section{Simulation Results and Discussion}

\subsection{Simulation Results}

The mathematical model was simulated using the Python odeint solver with the use of the nominal values given in Appendix. Different number of volume compartments were tested to find the lowest number of volume compartments which sufficiently represents the system, and 150 volume compartments are selected. To obtain the oscillatory behavior of the temperature, the inlet temperature to the heat exchanger $\left(T_{i}\right)$ was stepped down from $350^{\circ} \mathrm{C}$ to $230^{\circ} \mathrm{C}$.

The temperature transient for 150 volume compartments is shown in Figure 3, depicting the change of temperature as uniform oscillations at the exit of the reactor with time, when the feed temperature (the inlet to the heat exchanger) was stepped down by $120^{\circ} \mathrm{C}$. Initially, the reactor operated at steady state with a temperature of $350^{\circ} \mathrm{C}$. Then at $t=0.125 \mathrm{hr}$, the temperature is reduced by $120^{\circ} \mathrm{C}$. The system became unstable and showed oscillatory behavior. Temperature oscillations have a period of about 12 minutes and a maximum amplitude of about $320^{\circ} \mathrm{C}$.

When the feed temperature decreases, the temperature at the reactor inlet also decreases due to the decreased heat transfer. This will affect the temperature at the exit of the reactor due to two mechanisms, which are the direct heat transfer from the gas and the change of heat of reaction of the exothermic reaction. The latter is known to be faster than the former (Morud and Skogestad, 1993). Therefore at first, the rate of ammonia conversion decreases leading to an increase of reactant concentration and total number of molecules in the first few reactor compartments, which will decrease the outlet temperature of each reactor compartment. This can be seen from the number of moles and the outlet temperature transient of the volume compartments 1 and 5 in Figure 4. A sudden reduction of number of molecules with the temperature reduction can be observed for the volume compartments 10 and higher. This may be due to the combined effect of the faster reduction of $\mathrm{NH}_{3}$ molecules and slower increase of $\mathrm{N}_{2}$ and $\mathrm{H}_{2}$ 


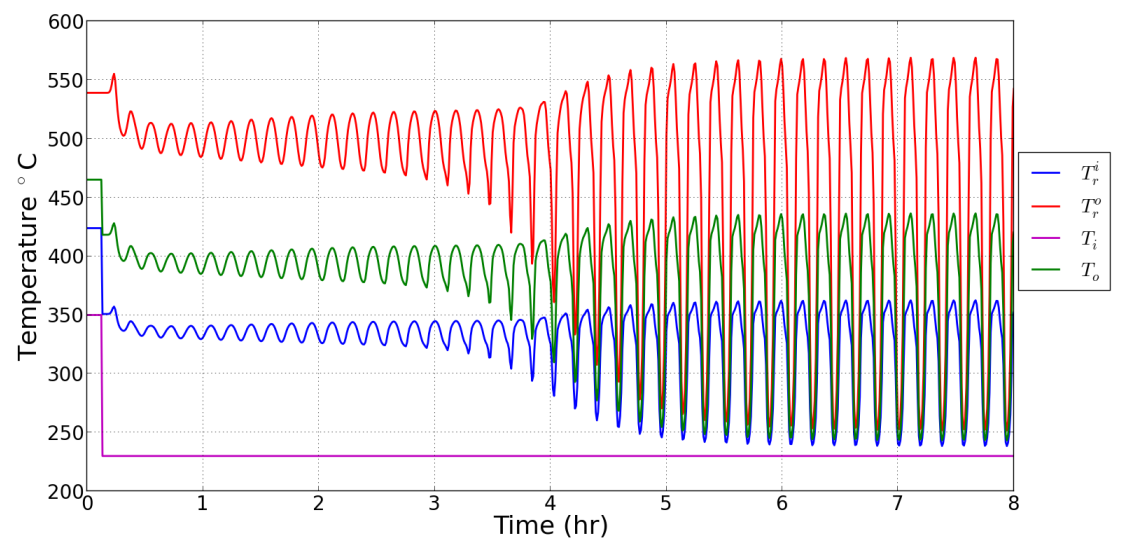

Figure 3. The temperature transient for 150 volume compartments, when a decrease in heat exchanger inlet temperature $\left(T_{i}\right)$ from $350^{\circ} \mathrm{C}$ to $230^{\circ} \mathrm{C}$ was done at $t=0.125 \mathrm{hr}$.

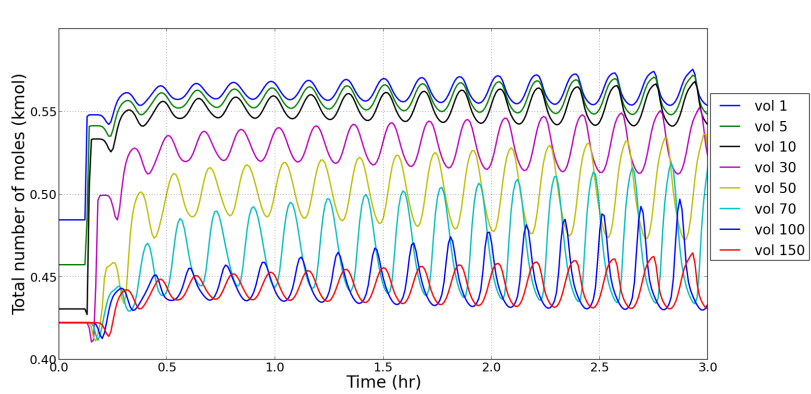

(a)

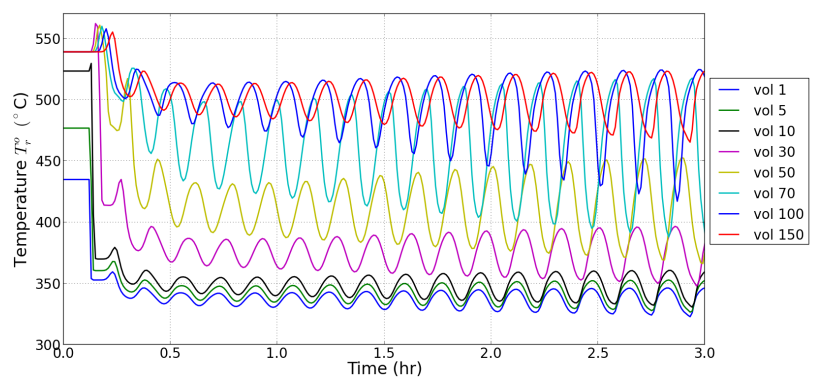

(b)

Figure 4. The total number of moles (4a) and the temperature (4b) with time, for different volume compartments along the reactor. Here 'vol' stands for the volume compartment number.

molecules. However, this gives a sudden increase to the exit temperature. The temperature of the reactor compartments along the reactor will increase due to the exothermic reaction of ammonia conversion. Therefore, the inlet temperature to the reactor will again increase by the heat transfer from the reactor exit streams. This dual effect of rate of reaction and the heat transfer will eventually result in an oscillatory behavior of number of moles in the reactor compartments leading to the same cyclic behavior in the outlet temperature of reactor compartments.

However, to obtain an optimum stabilized reactor per- formance, controlling of the temperature to the heat exchanger inlet will not be enough. The composition of feed gases, feed flow rate, feed temperature to the reactor inlet and the pressure along the reactor would be useful as monitoring measurements (Shah, 1967).

\subsection{Comparison with Previous Work}

The model developed by Naess et al. (1993) includes a reactor with three beds, an internal heat exchanger, an external heat exchanger, a compressor and a separator. A pressure drop is considered as a pressure drop across valves. Spatial discretization of states along the reactor beds is also done. Their model was verified using the plant data. However, the main objective was to test different control strategies.

The model used by Morud and Skogestad (1998) also consist of three beds in series with fresh feed make-up between each bed and pre-heating of feed with the effluent. Partial differential equations are used considering spatial discretization of temperature and the ammonia concentration in one direction. A dispersion coefficient is used for finding the finite heat transfer rate between the gas and the catalyst. The pre-heater is same as in this work, a steady state counter current heat exchanger, but the model used the Number of Transfer Units (NTU) approach with preheater efficiency. The temperature instability is obtained by changing the pressure of the reactor from 200 bar to less than 170 bar while the feed temperature was kept constant at $250^{\circ} \mathrm{C}$. It is stated that the same behavior could be observed by changing the temperature from $250^{\circ} \mathrm{C}$ to about $235^{\circ} \mathrm{C}$ while keeping the pressure constant at 200 bar, which is also observed in this work.

The reactor system used by Rabchuk (2014) and Rabchuk et al. (2014) consists of a reactor and a heat exchanger as in this study. This is due to the assumption that the temperature oscillations occur due to the reactor-heat exchanger system, which is proven true. The mole numbers of species in the reactor and the heat flow through the heat exchanger is kept as states unlike in previous models, 
where the concentration and temperature along the reactor beds were the states. The heat exchanger model includes dynamics and the Logarithmic Mean Temperature Difference (LMTD) approach with an approximation to the temperature difference. Instead of discretized partial differential equations, sets of ordinary differential equations have been used for 200 elementary volumes. The details of the reaction rate is not stated. Similar oscillatory behavior of temperature has been obtained by changing the temperature of the inlet to the system from $250^{\circ} \mathrm{C}$ to $200^{\circ} \mathrm{C}$.

The topology used in this work is similar to that of Rabchuk et al. (2014), and simpler than the topology of most other work. Only the number of moles in the reactor compartments are kept as states via species balances. The heat exchanger model is explicit with respect to the temperature, which simplifies the model compared to other work where an implicit model based on LMTD is used. Assuming ideal gas, and perfectly controlled pressure allows for eliminating the energy balance to compute the exit flow rates. All the data with values and units are welldocumented.

\section{Conclusions}

A mathematical model is developed for observing the dynamic behavior of an industrial ammonia synthesis reactor system which includes one reactor and a heat exchanger. All the data used in the simulation are taken from open literature and are presented in this work. The model is simple, but complete enough to satisfactorily reproduce the oscillatory behavior of the temperature of the reactor.

To obtain more accurate results, the model could be modified using the temperature dependent variables which are assumed as independent in this work and using more accurate catalyst activity values for the appropriate particle size of the catalyst.

\section{Acknowledgment}

Asanthi Jinasena thanks Anushka Perera for the help in learning Python.

\section{References}

R. F. Baddour, P. L. T. Brian, B. A. Logeais, and J. P. Eymery. Steady-state simulation of an ammonia synthesis converter. Chemical Engineering Science, 20(4):281-292, 1965. doi:10.1016/0009-2509(65)85017-5.

G. F. Froment, K. B. Bischoff, and J. D. Wilde. Chemical Reactor Analysis and Design. John Wiley \& Sons, 2nd edition, 2010.

L. J. Gillespie and J. A. Beattie. The Thermodynamic Treatment of Chemical Equilibria in Systems Composed of Real Gases, I. An Approximate Equation for the Mass Action Function Applied to the Existing Data on the Haber Equilibrium. Physical Review, 36:743-753, 1930.

J. Morud. Studies on the dynamics and operation of integrated plants. PhD thesis, University of Trondheim, 1995.
J. Morud and S. Skogestad. The Dynamics of Chemical Reactors with Heat Integration. In AIChE Annual Meeting, pages 115, St. Louis, 1993.

J. C. Morud and S. Skogestad. Analysis of instability in an industrial ammonia reactor. AIChE Journal, 44(4):888-895, 1998. doi:10.1002/aic.690440414.

A. Murase, H. L. Roberts, and A. O. Converse. Optimal Thermal Design of an Autothermal Ammonia Synthesis Reactor. Industrial \& Engineering Chemistry Process Design and Development, 9(4):503-513, 1970. doi:10.1021/i260036a003.

L. Naess, A. Mjaavatten, and J.-O. Li. Using dynamic process simulation from conception to normal operation of process plants. Computers \& Chemical Engineering, 17(5-6):585600, 1993. doi:10.1016/0098-1354(93)80046-P.

K. Rabchuk. Stability map for ammonia synthesis reactors. Master's thesis, Faculty of Technology, Telemark University College, Norway, 2014.

K. Rabchuk, B. Lie, A. Mjaavatten, and V. Siepmann. Stability map for Ammonia Synthesis Reactors. In 55th Conference on Simulation and Modeling (SIMS 55), pages 159-166, 2014.

M. Shah. Control Simulation in Ammonia Production. Industrial \& Engineering Chemistry, 59(1):72-83, 1967. doi:10.1021/ie50685a010.

C. P. P. Singh and D. N. Saraf. Simulation of ammonia synthesis reactors. Industrial \& Engineering Chemistry Process Design and Development, 18(3):364-370, 1979. doi:10.1021/i260071a002.

V. B. Singh. Modelling of Quench Type Ammonia Synthesis Converters. Master's thesis, Department of Chemical Engineering, Indian Institute of Technology Kanpur, India, 1975.

C. van Heerden. Autothermic Processes, Properties and Reactor Design. Industrial \& Engineering Chemistry, 45(6):12421247, 1953. doi:10.1021/ie50522a030. 


\section{Appendix: Data}

Parameters and operating conditions used for the simulation:

\section{Parameters}

A Heat transfer area (Morud, 1995)

$\tilde{c}_{p} \quad$ Molar heat capacity of gas mixture (Morud, 1995)

$\hat{c}_{p, c} \quad$ Specific heat capacity of catalyst (Morud, 1995)

$C_{p, c} \quad$ Total heat capacity of catalyst $m_{c} \hat{c}_{p, c}$

$\Delta H_{r} \quad$ Enthalpy of the reaction (Rabchuk et al., 2014)

$E_{-} \quad$ Activation energy of reverse reaction (Murase et al., 1970; Morud and Skogestad, 1998)

$\varepsilon \quad$ Void fraction of catalyst (Rabchuk et al., 2014)

$f$ Catalyst activity factor (Morud and Skogestad, 1998)

$k_{-}^{0} \quad$ Pre-exponential factor of reverse reaction (Murase et al., 1970; Morud and Skogestad, 1998)

$m_{c} \quad$ Total mass of catalyst $\rho_{c} V$

$M_{\mathrm{Ar}} \quad$ Molar mass of Ar atom

$M_{\mathrm{H}_{2}} \quad$ Molar mass of $\mathrm{H}_{2}$ molecule

$M_{\mathrm{N}_{2}} \quad$ Molar mass of $\mathrm{N}_{2}$ molecule

$M_{\mathrm{NH}_{3}} \quad$ Molar mass of $\mathrm{NH}_{3}$ molecule

$N \quad$ Number of reactor compartments (Decided after a few trials)

$v \quad$ Stoichiometric matrix

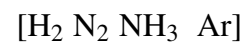

$p^{\sigma} \quad$ Atmospheric pressure

$R \quad$ Universal gas constant

$\rho_{c} \quad$ Packing density of catalyst (Rabchuk et al., 2014)

$U \quad$ Overall heat transfer coefficient (Morud, 1995)

$V \quad$ Volume of the reactor $57.2 \mathrm{~m}^{3}$ (Rabchuk et al., 2014)

\section{Operating Conditions}

$\dot{m}_{i} \quad$ Mass flow rate - reactor inlet (Rabchuk et al., 2014)

$p \quad$ Controlled reactor pressure (Rabchuk et al., 2014)

$T_{i} \quad$ Feed temperature (heat exchanger inlet) (Rabchuk et al., 2014)

$x_{\mathrm{H}_{2}}^{i} \quad$ Mole fraction of $\mathrm{H}_{2}$ at reactor inlet (Rabchuk et al., 2014)

$x_{\mathrm{N}_{2}}^{i} \quad$ Mole fraction of $\mathrm{N}_{2}$ at reactor inlet (Rabchuk et al., 2014)

$x_{\mathrm{NH}_{3}}^{i} \quad$ Mole fraction of $\mathrm{NH}_{3}$ at reac$125840 \mathrm{~kg}$ $39.95 \frac{\mathrm{kg}}{\mathrm{kmgl}}$ $2.016 \frac{\mathrm{kg}}{\mathrm{kmol}}$ $28.02 \frac{\mathrm{kg}}{\mathrm{kmol}}$

$17.034 \frac{\mathrm{kg}}{\mathrm{kmol}}$

150

$\left[\begin{array}{llll}-3 & -1 & 2 & 0\end{array}\right]$

$1.01325 \times 10^{5} \mathrm{~Pa}$ $8314 \frac{\mathrm{J}}{\mathrm{kmol} \cdot \mathrm{K}}$

$2200 \frac{\mathrm{kg}}{\mathrm{m}^{3}}$

$1.9296 \times 10^{6} \frac{\mathrm{J}}{\mathrm{h} \cdot \mathrm{m}^{2} \cdot \mathrm{K}}$ tor inlet (Rabchuk et al., 2014)

$67.6 \frac{\mathrm{kg}}{\mathrm{s}}$

$178 \times 10^{5} \mathrm{~Pa}$

$350{ }^{\circ} \mathrm{C}$

0.6972

0.24

0.0212 